Itinéraires Itinéraires

Littérature, textes, cultures

2019-1 | 2019

La « renaissance littéraire » africaine en débat

\title{
Passeurs de mémoire populaire : Kwani Trust et les lieux de l'histoire
}

Brokering Popular Memory: Kwani Trust and the Location of History

\section{Kate Wallis}

Traducteur : Aurélie Journo

\section{OpenEdition \\ Journals}

Édition électronique

URL : http://journals.openedition.org/itineraires/5947

DOI : $10.4000 /$ itineraires.5947

ISSN : 2427-920X

Éditeur

Pléiade

\section{Référence électronique}

Kate Wallis, «Passeurs de mémoire populaire : Kwani Trust et les lieux de l'histoire », Itinéraires [En ligne], 2019-1 | 2019, mis en ligne le 26 juillet 2019, consulté le 21 décembre 2020. URL : http:// journals.openedition.org/itineraires/5947 ; DOI : https://doi.org/10.4000/itineraires.5947

Ce document a été généré automatiquement le 21 décembre 2020.

\section{c.) (i)}

Itinéraires est mis à disposition selon les termes de la licence Creative Commons Attribution - Pas d'Utilisation Commerciale - Pas de Modification 4.0 International. 


\title{
Passeurs de mémoire populaire : Kwani Trust et les lieux de l'histoire
}

\author{
Brokering Popular Memory: Kwani Trust and the Location of History
}

\author{
Kate Wallis
}

Traduction : Aurélie Journo

\section{NOTE DE L'ÉDITEUR}

Ce texte est la traduction d'un chapitre d'une monographie à paraître et issue d'une thèse soutenue à l'université du Sussex en 2016, intitulée Literary Networks and the Making of 21st Century African Literature in English: Kwani Trust, Farafina, Cassava Republic Press and the Production of Cultural Memory.

Dans la nouvelle d'Andia Kisia "A Likely Story », le professeur Kimani, après avoir été emprisonné, torturé et contraint à l'exil pour ses textes sur Dedan Kimathi, est invité à rentrer au Kenya pour mener les recherches visant à retrouver la sépulture du chef des combattants Mau Mau (Kisia 2003). On ne connaît le narrateur qu'à travers son lien au professeur Kimani : il s'agit d'un collègue universitaire et compagnon de beuverie qui est soulagé de voir le professeur contraint de partir, car cela lui évite d'avoir à «le quitter ${ }^{1}$ " (83). À son retour au Kenya, cet « ami » l'accueille à l'aéroport, puis, un an après la mort $\mathrm{du}$ professeur lui rend le « petit service » de raconter l'histoire de son échec à retrouver des ossements qui auraient pu soutenir la nation et rendre justice au travail de sa vie.

«A Likely Story » a d'abord paru dans le premier numéro de la revue Kwani?, lancée en 2003 par la maison d'édition et collectif littéraire Kwani, basé à Nairobi. À travers ce récit, Kisia attire l'attention du lecteur sur le manque de fiabilité de l'histoire et de la mémoire, et sur les multiples façons dont, au Kenya, le passé a été effacé, approprié ou construit. La valeur et le sens de l'histoire en tant que discipline universitaire sont remis en question: le professeur Kimani se barricade derrière ses livres, et sa quête visant à écrire et à localiser Kimathi devient une telle obsession qu'il en perd sa famille, et ce, bien que, 
quarante ans plus tard, cette quête n'ait produit que peu de résultats (74), le sujet de sa recherche demeurant «impénétrable, énigmatique, caché sous une carapace de vérités " (76). Le narrateur montre comment la vision qu'a le professeur Kimani de Kimathi se construit en retirant « les coïncidences, les diverses imperfections (quoiqu'humaines) et tout autre fait gênant ${ }^{2}{ }$ et souligne que si le professeur a écrit des romans qui sont lus par des centaines de milliers de lecteurs à l'étranger, il n'est pas reconnu au Kenya par ceux qu'il considère comme son lectorat principal (82). Kisia remet en question non seulement la valeur et la fiabilité du travail de l'historien, mais aussi les sources primaires et secondaires sur lesquelles se construit toute narration historique. Les murs des archives nationales sont ainsi décorés des «falbalas de l'oubli»: on y trouve des photographies jaunies d'hommes politiques assassinés avec « les dates de naissance et de mort de chacun, avec un flou commode quant à la méthode (d'expédition) » (77). Kisia crée ainsi l'image percutante de l'histoire d'une nation qui a non seulement été négligée, mais qui a également fait l'objet de dissimulation et de manipulation. En témoigne l'image des archivistes qui font disparaître les « textes qui dérangent » en les avalant, ne laissant derrière eux que des pages "à moitié mâchées" et indigestion (80-81). Quand le professeur Kimani et le narrateur essaient de trouver ce qu'ils cherchent en dehors des archives nationales, dans des récits oraux, leur source leur indique qu'il « en avait assez qu'on lui creuse la tête et qu'on transforme ce qu'on en extrayait en récits qu'il ne reconnaissait plus, lui qui les avait racontés ${ }^{3} »(81)$.

3 La nouvelle, dont l'action se situe en 2003, année de sa publication et des célébrations des quarante ans d'indépendance du Kenya, s'attache à disséquer la relation particulière qui unit le passé et la construction de l'idée nationale au cours de ces quarante années. Le texte de Kisia montre une nation construite à la fois sur l'appel à l'amnésie historique défendu par son premier président Jomo Kenyatta pour unir le pays («oublier et pardonner", 78) et sur toute une série de "monuments mal bâtis à la gloire de sa complaisance " érigés par Kenyatta qui plantaient « les germes de son propre souvenir » en donnant son nom aux bâtiments et aux rues à travers le pays (76). Les recherches entreprises par le nouveau gouvernement pour retrouver la sépulture de Kimathi et autour desquelles se construit la narration, sont présentées comme un acte de mémoire alors même qu'elles s'intègrent à une entreprise plus large d'effacement de la mémoire, manière de « rediriger la bile collective vers d'autres cibles » (82). De manière frappante, personne ne sort innocent de ce processus et le narrateur avance l'argument d'une responsabilité collective dans l'écriture de l'histoire et la création de héros: «De cela, nous sommes complices. Nous autorisons l'imagination à l'emporter sur les faits » (77).

La nouvelle d'Andia Kisia et le fait qu'elle a été publiée dans le premier numéro de la revue Kwani? met en lumière ce qui nous intéresse ici, à savoir la façon dont le Kwani Trust a œuvré à créer de nouveaux lieux et de nouvelles formes d'expression de la mémoire culturelle. La ligne éditoriale et les textes des principaux auteurs du Kwani Trust (comme Andia Kisia, Parselelo Kantai, Billy Kahora, Yvonne Adhiambo Owuor et Binyavanga Wainaina) témoignent d'une préoccupation particulière pour l'historiographie, et pour les façons dont la mémoire et l'histoire se construisent et prennent forme en tant que textes. En replaçant ces questions dans le contexte plus large de la production culturelle au Kenya, et notamment celle financée par les donateurs étrangers et qui s'intéresse, elle aussi, aux questions d'histoire et de mémoire, nous aimerions rendre compte des différentes manières dont la revue Kwani? aborde et explore les questions que soulève avec force la nouvelle de Kisia: Où se situe la production du 
savoir historique au Kenya? Qui produit l'histoire et pour qui ? Quels sont les liens qui unissent mémoire, littérature et matérialité ? Nous ferons dialoguer la notion de mémoire collective, élaborée par l'égyptologue Jan Assmann et constituée selon lui de la « mémoire culturelle » et de la «mémoire communicative », avec les définitions que propose Karin Barber des «arts populaires» (Barber 1987) afin de montrer comment ce groupe d'écrivains s'attache à la construction d'une mémoire populaire.

\section{Construction de la mémoire}

5 L'égyptologue allemand Jan Assmann a développé le concept de "mémoire culturelle » afin de nuancer la notion de "mémoire collective » développée par Maurice Halbwachs. Le travail fondateur de ce dernier avait rendu visible la façon dont les individus «se souviennent, reconnaissent et situent leurs souvenirs " au sein de cadres socialement déterminés (Halbwachs 1992: 43). Reprenant les travaux de Halbwachs, Assman avance que la mémoire collective est constituée de deux formes de mémoire qui s'entrecroisent: la mémoire communicative et la mémoire culturelle (Assmann 1995). La mémoire communicative se définit par son inscription dans la «communication quotidienne». Assmann souligne que si ces échanges sont régis par des conventions, la mémoire communicative se caractérise quant à elle par des traits de "non-spécialisation, de réciprocité des rôles, d'instabilité thématique et de désorganisation ${ }^{4}$ » (Assmann 1995 : 126). Un autre trait distinctif de la mémoire communicative est selon lui de se situer dans un horizon temporel limité, difficilement localisable dans «le passé toujours en expansion du passage du temps" (Assmann 1995: 129). Au contraire, la mémoire culturelle se définit par son ancrage dans des points de référence fixes dans le passé et renvoie à la façon dont la mémoire d'une époque ou d'un événement particuliers est entretenue par le biais de formations culturelles ou de communication institutionnelle, un processus au cœur duquel se situent les notions de "formation » et d'" organisation ». Assmann définit la mémoire collective comme une « culture objectivée » : un ensemble de «textes, d'images, de rites, de bâtiments, de monuments, de villes, ou même de paysages » à travers lequel une société donnée construit son identité et son rapport au passé (Assmann 1995 : 128). C'est dans son lien à un sentiment d'identité collective - lien également visible dans la mémoire communicative - que la mémoire collective se distingue de l'histoire (Assmann 1995 : 128). Assman insiste sur les dynamiques à l'œuvre dans la mémoire culturelle, dynamiques qui se manifestent différemment selon le lieu et l'époque, mais dans lesquelles les processus de «transformation et de transmission » sont centrales, particulièrement entre mémoire communicative et mémoire culturelle. Ce qui suit cherche donc à montrer que ces processus de transformation et de transmission jouent un rôle essentiel dans la façon dont les textes littéraires publiés par le Kwani Trust interviennent dans la «formation » et l'« organisation » de la mémoire culturelle, en ce que ceux-ci s'appuient sur les distinctions opérées par Assmann entre mémoire culturelle et mémoire communicative comme deux façons de construire la mémoire, tout en problématisant ces distinctions.

6 Afin d'analyser la façon dont le Kwani Trust a pu créer de nouveaux lieux et de nouvelles formes d'expression de la mémoire culturelle, il est nécessaire de replacer le travail de ses membres sur l'histoire et la mémoire dans une conversation plus large autour de ces questions. Depuis la création du Kwani Trust en 2003, les débats médiatiques et universitaires témoignent d'une préoccupation particulière pour ce que Dan Ojwang et 
Lotte Hughes ont respectivement appelé "une crise de l'historicité dans la culture politique kenyane » (Ojwang 2009: 36) et « une crise de la mémoire collective » (Hughes 2011 : 183). Par exemple, dans un article universitaire publié en 2003 et intitulé «Mau Mau \& the Contest for Memory ", Marshall S. Clough analyse le caractère insaisissable de la mémoire autour des Mau Mau et la manipulation dont celle-ci a fait l'objet, comme le fait Kisia dans « A Likely Story ». Il décrit la façon dont Kenyatta a soutenu une "politique d'amnésie » et analyse la « crise de la mémoire » qui s'ensuivit (Clough $2003: 256,268$ ). À propos de sa recherche menée dans le cadre du projet «Managing Heritage, Building Peace: Museums, memorialisation and the uses of memory in Kenya » financé par l'AHRC (Arts and Humanities Research Council, 2008-2011), Lotte Hughes souligne que cette « amnésie orchestrée » s'étend au-delà de la lutte pour l'indépendance :

[et] continue à recouvrir une variété de sujets, comme le régime tyrannique du président Moi (1978-2002) et son héritage, les assassinats politiques, le recours à l'emprisonnement et à la torture sous les régimes de Kenyatta et de Moi, l'accaparement des terres par les élites, la corruption extravagante, etc. ${ }^{5}$. (Hughes $2011: 187)$

Elle souligne de façon significative que la plupart des Kenyans le savent et que «cela fait l'objet de débats quasi-quotidiens dans les médias » (Hughes 2011: 187), débats qui se sont intensifiés après la vague de violences qui suivit les élections présidentielles kenyanes de 2007. Dans cet article, et en écho à la nouvelle de Kisia, Hughes attire notre attention sur la façon dont la répression de la mémoire se poursuit parallèlement à une «mémorialisation florissante autour des Mau Mau » (Hughes 2011 : 183, 186).

7 La nouvelle de Kisia, «A Likely Story », et le premier numéro de la revue Kwani? furent publiés juste après l'élection de Mwai Kibaki, dirigeant du parti de la National Rainbow Coalition (NARC), à la présidence en décembre 2002. Son prédécesseur, Daniel arap Moi était resté au pouvoir vingt-quatre ans et sa présidence était devenue synonyme d'une époque de corruption et de répression politique. La revue fut ainsi lancée à un moment où les électeurs kenyans étaient plein d'espoir que l'« ouverture et l'unité » que représentait le nouveau gouvernement NARC soient «une occasion de mener un changement véritable» (Branch 2011: 249), en un mot dans «un contexte de libération et de transition politique » comme le soulignent les auteurs de l'introduction à l'ouvrage Rethinking Eastern African Literary and Intellectual Landscapes (Ogude, Musila et Ligaga 2011a: xi). Il est significatif que la revue commence à être publiée à un moment où le nouveau régime politique donnait des gages quant à la possibilité que les discours sur le passé, et plus particulièrement sur la répression et la manipulation de celui-ci, puissent sortir des espaces populaires ou informels où ils circulaient jusqu'alors pour intégrer des espaces publics plus formels. James Ogude (2007) et Joyce Nyairo ont par exemple tous les deux montré comment, en opérant «en dehors des espaces de contrôle officiel», la musique populaire avait pu représenter un espace dans lequel, depuis l'indépendance, l'histoire kenyane pouvait être décrite et où des «lectures radicalement différentes du passé » pouvaient s'exprimer (Nyairo 2005). La négociation de cette transition, ou pour reprendre le vocabulaire d'Assmann de cette "transmission " entre culture populaire kenyane et forme littéraire fut ainsi au cœur de l'intervention opérée par le Kwani Trust sur la mémoire culturelle. Que la fin du régime Moi ait été synonyme d'ouverture des espaces publics à la circulation des discours, ainsi que de la possibilité pour la production culturelle de s'intéresser au passé, est visible non seulement dans la création de la revue mais également dans la renaissance de toute une série d'initiatives en matière de protection du patrimoine portées par la société civile (Hughes, Coombes et Karega- 
Munene 2011 : 176), dans la création d'un espace artistique pluridisciplinaire, le GoDown Arts Centre en 2003 à Nairobi (GoDown Arts Centre 2016) ou encore dans la fondation de Ketebul Music en 2007, dont le but est de décrire et d'archiver la musique produite dans la région « au cours des soixante dernières années ${ }^{6}$ " (Ketebul Music 2016).

Comme l'indique Hughes, la nature des débats autour de la mémoire et du passé au Kenya a changé et ces débats se sont intensifiés à mesure que l'euphorie de 2002 cédait la place à la désillusion et quand l'élection présidentielle de 2007 donna lieu à des violences interethniques qui entraînèrent la mort de plus d'un millier de personnes et le déplacement interne de plus de 400000 autres (Njogu 2009b : 2). Ces événements donnèrent lieu à toute une série de nouvelles conversations, qui pour beaucoup furent financées par la Fondation Ford : une série bimensuelle de débats à l'Institut Goethe modérés par Mbugua wa Mungai et George Gona et intitulée «(Re)membering Kenya » mais aussi une variété de projets et de publications menés par Twaweza Communications, une maison d'édition basée à Nairobi et fondée par le professeur Kimani Njogu. Twaweza publia ainsi les trois volumes de la série de livres intitulée (Re)membering Kenya ainsi que d'autres ouvrages comme Healing the Wound: Personal Narratives About the 2007 Post-Election Violence in Kenya (Njogu 2009a) et Defining Moments: Reflections on Citizenship, Violence and the 2007 General Elections in Kenya (Njogu 2011b). Njogu décrit sa motivation comme ancrée dans une double conviction : celle qu'il ne fallait pas laisser l'État être la seule instance autorisée à interpréter les événements et celle de l'importance de prendre en compte "les sources peu recherchées » dans lesquelles se laissent lire une variété de voix et de points de vue alternatifs (Njogu 2011a). Le double numéro cinq de la revue Kwani? témoigne de motivations similaires. Il proposait une réponse aux violences post-électorales et était présenté comme "un récit collectif de ce que nous étions avant, et de ce que nous sommes devenus au cours de l'épisode historique des cent premiers jours de $2008^{7}$ " (Kahora 2008a). Il faut ajouter ici que Binyavanga Wainaina, le rédacteur-fondateur du Kwani Trust, et Yvonne Adhiambo Owuor, dont la nouvelle « Weight of Whispers », paru dans le premier numéro de la revue, a remporté le prix Caine en 2003, ont tous les deux réfléchi à la façon dont les violences post-électorales ont informé leurs premiers romans respectifs One Day I Will Write About This Place (Wainaina 2014) et Dust (Owuor 2013).

En replaçant le Kwani Trust dans un contexte culturel particulier, dans lequel la production culturelle financée par des donateurs étrangers se concentre sur les questions d'histoire et de mémoire, selon des modalités différentes en 2002 et en 2007, souligner la novation littéraire du Kwani Trust ou s'interroger sur les raisons de sa préoccupation pour l'histoire paraît insuffisant. Il s'agit plutôt de voir ici quelle est la particularité de la contribution du Kwani Trust et de la littérature dans la médiation et la forme de la mémoire culturelle au sein de cet environnement culturel plus large.

\section{Prendre forme, trouver une forme : le Kwani Trust}

10 La recherche de Macharia Mwangi a montré la volonté de Kwani Trust de «consigner l'histoire politique et sociale du Kenya " (199) et la façon dont la revue Kwani? a abordé différentes facettes de l'histoire kenyane (227) en rassemblant et en publiant des documents et des textes qui traitent en particulier des sociétés précoloniales, des archives du mouvement Mau Mau, de l'histoire politique après Kenyatta, et des violences politiques de 2008 (Mwangi 2015). Prolongeant cette réflexion, nous avançons l'idée que 
le travail du Kwani Trust sur l'histoire et la mémoire au Kenya se fait par la mise en relation de trois formes de textes.

Tout d'abord, plusieurs écrivains étroitement associés au Kwani Trust, et légitimés par celui-ci, parmi lesquels Andia Kisia, Billy Kahora, Parselelo Kantai et Yvonne Adhiambo Owuor, semblent avoir vu en la forme fictionnelle un outil permettant de dévoiler des histoires jusqu'alors cachées et d'explorer la façon problématique dont les récits historiques officiels se construisent au Kenya. Andia Kisia, par exemple, a vu ses textes publiés dans cinq des huit numéros de Kwani? parus à ce jour, et depuis 2005, son parcours est mis en avant sur le site du Kwani Trust : après avoir été publiée dans Kwani? elle a en effet obtenu une bourse auprès du Royal Court Theatre de Londres (Kwani Trust 2005). Après la publication de "A Likely Story » trois de ses textes furent publiés dans le deuxième numéro de la revue (Wainaina and Kalondo 2004). Ces trois textes éclairent de différentes manières les silences qui demeurent dans les récits historiques kenyans : sa nouvelle « 1982 », dont le titre fait référence à la tentative de coup d'État militaire de la même année, raconte l'histoire d'un policier en charge du registre officiel qui y consigne (avant de se les approprier) les vêtements et effets personnels des personnes arrêtées suite à ce coup d'État manqué (7-24). De la même façon, les textes d'Yvonne Adhiambo Owuor témoignent de sa volonté de mettre en lumière les archives mémorielles qui existent au Kenya. Owuor fait partie du groupe d'écrivains à l'origine de l'idée grâce à laquelle est né le Kwani Trust, et sa nouvelle "Weight of Whispers » fut publiée dans le premier numéro de la revue. Son premier roman, Dust, fut publié dix ans plus tard, et sa sortie fut intégrée aux festivités liées aux dix années d'existence du Kwani Trust. Comme la nouvelle de Kisia " A Likely Story ", le roman Dust s'intéresse aux effets destructeurs de l'héritage de Jomo Kenyatta et de son appel à garder le silence sur les difficultés du passé pour construire un Kenya indépendant et uni. Dans Dust on voit le patriarche Aggrey Nyipir Oganda et ceux de sa génération se débattre avec ce silence, et « chanter à pleine voix l'hymne national » avec l'espoir vain qu'il pourra ainsi protéger ses enfants des regrets, silences et trahisons du passé et du présent (Owuor 2013 : 30). Dans la scène qui ouvre le roman, le fils de Nyipir, Odidi est abattu par la police dans les rues de Nairobi, avec en toile de fond les violences post-électorales de 2007-2008. Cet événement sert de point de départ à l'élucidation des silences et des histoires cachées à laquelle procède le roman. Aux yeux de Nyipir, le meurtre de son fils par l'État est directement lié à une histoire plus longue de mort et à ses espoirs déçus en la jeune nation kenyane. Les textes de Parselelo Kantai soulèvent des questions similaires : où est produit le savoir au Kenya, qui écrit l'histoire ? Kantai fut également l'un des membres fondateurs du Kwani Trust, et comme Kisia, il est fait référence à son succès sur le site du collectif et dans le deuxième numéro de la revue, notamment au fait qu'une de ses nouvelles a été nominée pour le prix Caine en 2004. Cette nouvelle, intitulée «Comrade Lemma \& the Black Jerusalem Boys Band» (Kantai 2004) retrace les aventures de Comrade Lemma, qui fut dans sa jeunesse le chanteur principal d'un groupe populaire du quartier et le compositeur de Joka, une chanson d'abord associée au mouvement de libération avant d'être interdite au cours de la première année d'indépendance du pays. À la veille des célébrations du quarantième anniversaire de l'indépendance, l'entreprise de préservatifs Careful Love condoms le « retrouve " vivant dans l'anonymat et la pauvreté, et il est célébré en héros national, malgré une série d'erreurs sur son identité. Tout au long de la narration, Kantai attire subtilement l'attention du lecteur sur les différentes façons dont l'identité de Comrade Lemma, le narrateur et sa chanson Joka font l'objet de réécritures et 
d'appropriations. Dans un article paru en 2006 dans The East African, Kantai affirmait la nécessité que le savoir historique sur l'Afrique soit produit sur le continent et non plus par les universitaires occidentaux, arguant que «ceux qui importent leurs histoires se condamnent par l'échec de leur propre imagination ${ }^{8}$ " (Kantai 2006 : 249). Il conclut en affirmant que la réappropriation du «territoire» de l'histoire représente «la nouvelle frontière pour l'intellectuel africain» (Kantai 2006: 249). Sa fiction, présentée comme " une tentative de réengager le dialogue_avec l'histoire kenyane officielle ou publique » (Kantai 2011), peut être lue comme une réponse ou une "contribution» (Musila 2014 : 250) à ce défi.

12 En second lieu, en tant qu'institution, le Kwani Trust s'est efforcé de soutenir et de promouvoir les recherches et l'écriture de textes s'intéressant aux récits non fictionnels sur des personnes, des événements ou des lieux jusqu'alors peu représentés en littérature ou sous une forme imprimée. On trouve par exemple dans le premier numéro de la revue (Wainaina 2003b) un texte intitulé "The Life and Times of Richard Onyango »-sorte d'éducation sentimentale et artistique d'un des artistes kenyans les plus connus, telle que l'artiste l'a racontée à Binyavanga Wainaina, et qui se concentre sur sa relation avec Drosie, source d'inspiration de son œuvre (174-209). Le premier livre publié en dehors de la revue est une commande du Kwani Trust : il s'agit des mémoires, intitulés Kizuizini ( en détention ») et rédigées en swahili, de Joseph Muthee, ancien prisonnier des camps de détention britanniques pendant la révolte des Mau Mau (Muthee 2006). À ces mémoires succéda le récit non fictionnel de Billy Kahora, The True Story of David Munyakei (Kahora 2008c), dont une partie avait déjà été publiée dans le troisième numéro de la revue (Kahora and Wainaina 2005) et qui s'attache à faire le récit, jamais encore entrepris, de la trajectoire du lanceur d'alerte dans le scandale Goldenberg, dont les actes permirent de sauver l'économie kenyane de la faillite mais qui termina sa vie dans la pauvreté et l'anonymat. Ce texte, comme la nouvelle de Kantai, met en lumière les intérêts politiques et commerciaux qui sous-tendent la construction de héros nationaux. Par le biais de la forme littéraire adoptée dans ce texte, Kahora entreprend de consigner et de conserver une trace de la vie de David Munyakei. Il souligne également, à travers la variété des perspectives et des formes intégrées au texte, que pour saisir une version de la vérité il est nécessaire de donner à voir la nature changeante et multiple de son sujet. Kahora devint rédacteur en chef de Kwani? en 2008 et s'attacha alors à promouvoir la création non fictionnelle comme genre à même de documenter les élections de 2007 dans le cinquième numéro de la revue (Kahora 2008a, b). Les textes non fictionnels publiés par le Kwani Trust, comme Kizuizini et Tale of Kasaya - mémoires d'une jeune domestique, Eva Kasaya, qui montrent ce qui se passe « derrière les portes fermées de la famille urbaine kenyane et révèlent des espaces que nous reconnaissons tous, mais dont nous refusons d'admettre qu'ils existent ${ }^{9}$ » (Kwani Trust 2016) - furent salués par la critique et reçurent tous deux le prix littéraire Jomo Kenyatta, décerné tous les deux ans par la Kenya Publishers Association.

13 Enfin, de façon complémentaire à la création non fictionnelle mise en avant pour documenter l'histoire politique, la vie quotidienne et la production culturelle, les premiers numéros de Kwani? comprenaient une grande variété de documents issus de la culture populaire kenyane, comme des entretiens, des échanges de courriels, des vieilles photographies, ou encore des paroles de chansons, qui jusqu'alors n'avaient jamais été rendues visibles dans un ouvrage imprimé. Le premier numéro de la revue, par exemple, comprend une série de photographies de Marion Kaplan intitulée «Scenes from the 
Past » et dans laquelle on trouve des images du premier vice-président kenyan et futur meneur de l'opposition, Oginga Odinga, prises lors d'un rassemblement politique en 1966, ou de Tom Mboya dans son cercueil après son assassinat politique en 1969 (Wainaina 2003b : 48-50). À ces photos succède dans la revue un entretien de Binyavanga Wainaina avec le groupe de hip-hop Kalamashaka, qui à l'époque bouleversait la scène musicale de Nairobi et dépassait les divisions de classe entre est et ouest de la ville grâce à leur rap en sheng qui mêlait "paroles percutantes et message poétique de justice sociale " (Wainaina $2003 \mathrm{~b}$ : 54) tout en se tournant vers le passé pour défendre le changement : «Dis-moi qui le premier prononcerait le mot harembee? Dedan Kimathi ou Mzee Jomo Kenyatta ${ }^{10}$ ?» (Kalamashaka 2003). Ce numéro s'attaquait également à la façon dont les stéréotypes circulent dans les formes culturelles populaires : on y trouve reproduit un courriel qui circula largement au Kenya, intitulé «Vain Jango » (Wainaina 2003b: 103-112), et une réponse imaginée par Binyavanga Wainaina et Muthoni Garland. Le message original, écrit dans un mélange d'anglais, de swahili et de sheng vient d'un Luo anonyme, qui raconte sa rencontre avec une femme dans un bar de la capitale kenyane, et comment il est tombé sous le charme de son derrière ("serious bootie») avant que son accent rural Okuyu (Wainaina 2003b : 104, 106) ne le fasse changer d'avis. On trouve dans le premier numéro de Kwani? une reproduction du mail accompagné de la "réponse cinglante » (Musila 2014) de la femme en question, imaginée par Binyavanga Wainaina et Muthoni Garland. On retrouve cette inclusion d'une variété de formes issues de la culture populaire kenyane dans Kwani? 3, avec deux photographies: l'une de Dedan Kimathi (Kahora et Wainaina 2005 : 5) et l'autre d'un couple de jeunes mariés prise en 1963 au studio Venus sur la rue Victoria (aujourd'hui rue Tom Mboya) et accompagnée d'une légende indiquant que « de nombreux services proposés dans cette rue ne furent ouverts aux Africains noirs qu'à partir de $1963 »$ (123). On y trouve également reproduites les paroles de chanson des membres du collectif Ukoo Flani Mau Mau (dont faisait partie Kalamashaka) et qui sont présentées comme de la poésie en sheng (167-190) ainsi que des reproductions de pages et de couvertures datées de 1975 et 1976 du magazine populaire kenyan Joe.

14 C'est selon nous à travers l'interaction entre ces trois formes de textes - fiction, création non fictionnelle, et éléments extraits des archives ou de la production contemporaine de la culture populaire kenyane - que le Kwani Trust intervient dans la construction de la mémoire culturelle. Billy Kahora souligne à maintes reprises la volonté de Kwani? de s'intéresser aux objets qui «étaient absents des textes imprimés » et décrit le rôle du travail engagé par le Kwani Trust comme étant de «mettre en avant la mémoire populaire contre la mémoire officielle » (Stasio 2012). Les textes commandés par le Kwani Trust relèvent ainsi en partie de ce désir de documenter ou de rendre public toute une série de récits sur le passé ou le présent kenyans, récits qui étaient selon Kahora, absents « des textes publics officiels et formels » (Kahora 2007 : i). Toutefois, il nous semble que la ligne éditoriale du collectif et de ses écrivains témoigne d'une préoccupation pour l'historiographie plus que pour l'histoire. En effet, à travers ces publications, l'intention du Kwani Trust est moins peut-être de documenter que d'interroger et de mettre au jour la façon dont la mémoire et l'histoire sont construites et légitimées en tant que textes. En rassemblant une variété de formes issues de la culture populaire kenyane et en les publiant avec des formes littéraires fictionnelles, le Kwani Trust rend visibles les formes sous lesquelles ces histoires et récits considérés comme « cachés » existent déjà. 


\section{Passeurs de mémoire populaire} qui mettent en relation ces différentes formes populaires et littéraires. L'éditorial de Billy Kahora qui ouvre Kwani? 4 commence par ces mots : «Attends que je te raconte » (« Let me tell you... ») (Kahora 2007 : i). Cette phrase est le point de départ d'une réflexion de Kahora sur le Kenya comme espace où circulent les récits (" a storytelling space »), dans laquelle il décrit la façon dont il ne cesse de rencontrer « dans les bars, les matatus ${ }^{11}$, les églises, aux coins des rues » des individus qui engagent la conversation par ces mots «Attends que je te raconte... » avant de le tenir " captivé » (« spellbound ») par « une histoire incroyable de plus» (Kahora 2007: i). Il poursuit en suggérant que s'exprime dans ces espaces « une version de la vérité sur la plupart des questions nationales ou locales » et souligne que le choix du terme « incroyable » est délibéré :

Et même si les faits ont leur importance, c'est l'invitation à être ensemble, le sentiment que j'ai assez en commun avec un inconnu pour l'écouter, pour croire l'incroyable, la version la plus invraisemblable, c'est cela qui me fait me sentir kenyan plus que tout autre chose ${ }^{12}$. (Kahora 2007 : i)

Dans cet éditorial, Kahora avance que ces récits en disent certes beaucoup sur la nature de la condition kenyane, mais représentent surtout ce qui permit de la maintenir au cours des années « sombres » du régime Moi, alors que l'économie kenyane était affaiblie par le scandale Goldenberg (Kahora 2007 : i, iii). À deux reprises dans ce texte, il regrette que " ces récits ne trouvent jamais leur place dans nos textes publics officiels et formels », et insiste que ces mêmes récits sont la raison d'être de Kwani? (Kahora 2007: i, iii). L'historien E.S. Atieno Odhiambo a montré que le contrôle étroit maintenu sur les institutions publiques et l'absence d'espace où aurait pu s'exprimer une opposition politique constituée ont fait des espaces informels, comme les enterrements, les "républiques populaires des matatus", les organisations religieuses, le football, la musique populaire ou les bars, des lieux d'expression démocratique alternatifs (Odhiambo 1987 : 200-1). Ce sont précisément ces lieux, caractérisés par Odhiambo par la vigueur de la conscience politique qui s'y exprime (Odhiambo 1987 : 201), que Kahora identifie à des sites de circulation des récits et des vérités nationales ${ }^{13}$.

Comme l'ont souligné les ouvrages sur la culture populaire est-africaine publiés il y a une dizaine d'années (Nyairo 2007, Ogude et Nyairo 2007, Ogude, Musila et Ligaga 2011b), la situation mise en lumière par Odhiambo explique l'émergence au Kenya d'une scène artistique populaire foisonnante, caractérisée par sa créativité, son sens de l'innovation et son énergie, et qui représentait une des rares formes d'expression permettant de s'attaquer aux questions complexes du pouvoir à l'abri de la surveillance gouvernementale ${ }^{14}$ (Ogude, Musila et Ligaga 2011a : viii). Dans le tout premier éditorial de la revue, Binyavanga Wainaina prend acte de cette «énergie créative» des arts populaires en dressant une liste de ses manifestations, du hip-hop d'Ukoo Flani Mau Mau à l'exposition de l'artiste Joga dans les rues et ruelles d'Eastleigh et de Mathare (Wainaina 2003a: 6). Il décrit cette forme d'expression créative comme relevant d'une certaine "esthétique » et souligne que c'est au Kenya qui s'y exprime que s'intéresse Kwani? (Wainaina 2003a). L'idée que la revue cherche à s'inspirer de cette esthétique qui puise ses sources dans la culture populaire kenyane est mise en avant par Billy Kahora dans l'éditorial qu'il signe dans le troisième numéro. Il raconte comment le groupe d'écrivains réunis pour travailler à ce numéro a discuté de la façon dont leur écriture pourrait 
donner à voir « le langage de la rue kenyane, de la campagne kenyane, du bar kenyan, et bien sûr le langage de la famille kenyane ${ }^{15}$ ", leur " saint Graal » - inspiré en cela par des musiciens kenyans comme le collectif Ukoo Flani Mau Mau - étant d'« écrire comme les Kenyans parlent, vivent et respirent " (Kahora 2005 : 6). Ces trois éditoriaux mettent en avant la relation des rédacteurs en chef de Kwani? à la culture populaire kenyane, relation centrale dans la ligne éditoriale de la revue, avec la double volonté d'attirer l'attention sur les récits et histoires qui circulent dans ces lieux et de développer une esthétique et des formes littéraires qui s'en inspirent. Ce dernier point soulève des questions importantes - évoquées notamment par Grace Musila (2014) et Tom Odhiambo (2011) quant à la relation qu'entretiennent les rédacteurs en chef de Kwani? avec les catégories du « local » et du « populaire », et à la façon dont celle-ci s'articule à la volonté de ceux-ci d'établir des liens et de se faire connaitre au-delà des frontières nationales.

L'essai dans lequel Musila développe ces questions est intitulé « Archives of the Present in Parselelo Kantai's Writing» et fut publié dans l'ouvrage collectif dirigé par Stephanie Newell et Onookome Okome à l'occasion de la date anniversaire des 25 ans de la publication de l'essai fondateur de Karin Barber, «Popular Arts in Africa (Barber 1987). Cet essai sert de point de départ à Musila qui cherche à étudier la façon dont Kantai et ce qu'elle appelle la "génération Kwani? » «repoussent les frontières des arts populaires dans une multitude de directions" (Musila 2014: 246). Elle analyse la relation des écrivains de la génération Kwani? au «populaire " à travers l'éditorial de Binyavanga Wainaina dans Kwani? 1. Il y rend hommage à cette énergie créative des arts populaires au Kenya en l'associant à l'innovation et à la " nouveauté », deux éléments qui permettent le développement d'une esthétique qui :

[...] ne nous sera léguée ni par les couloirs de l'université, ni par le ministère de la culture ou le Centre Culturel Français. Elle viendra de la création individuelle de milliers d'individus créatifs ${ }^{16}$. (Wainaina 2003a: 6)

Musila lie la rhétorique à l'œuvre dans le texte de Wainaina à la définition que Barber donne des arts populaires comme non officiels et «libres d'opérer entre des systèmes culturels établis sans pour autant se plier à leurs conventions », et comme tenant leur "caractère novateur" de la manière dont ils "associent des éléments des cultures traditionnelles et métropolitaines de façon inédite, se différenciant radicalement de chacune d'entre elle $^{17} »$ (Barber 1987 : 13). Musila réinvestit le continuum entre tradition et élites développé par Barber pour mettre en lumière la façon dont le programme d'écriture de Kantai et des contributeurs de Kwani? plus généralement, «enjambe » la ligne de partage déjà floue qui sépare "canonique» et "populaire» (Musila 2014: 245-246). Elle évoque en particulier les liens qui unissent le Kwani Trust aux espaces littéraires de Londres, Paris, New York ou du Cap, sa position de projet de la classe moyenne nairobienne dont le public principal est cette même classe moyenne de Nairobi, ainsi que la façon dont il dépend pour ses activités du soutien de donateurs du Nord global. L'ensemble de ces éléments contribue à interroger les concepts de " populaire » et de "réseaux de relations» (Barber 1987:1) grâce auxquels émerge cette écriture qui « élargit les horizons de la pratique littéraire populaire au Kenya » (Musila 2014 : 251-252, 262-263).

Le travail de Musila démontre avec force que les travaux pionniers de Barber sur les arts populaires peuvent offrir des jalons intéressants pour tenter de resituer le Kwani Trust dans un réseau littéraire panafricain et dans l'espace littéraire mondial. Barber insiste sur la nécessité de ne pas envisager les catégories de traditionnel, de populaire et d'élitiste comme des «catégories empiriques de produits culturels» mais de les considérer au 
contraire comme des "champs d'expression " traversés par différentes tensions et des concentrations de styles d'expression variés (Barber $1987: 19$ ). Stephanie Newell illustre ce point quand elle montre que, dans le cas des romans ouest-africains, si l'on se place dans la perspective du lectorat local, la distinction entre « élitiste » et " populaire » est peu opératoire, et que l'on perd de vue la "complexité du discours littéraire africain » si l'on cherche à "définir les textes "littéraires" contre et par rapport aux romans "populaires" » (Newell 2000 : 158). En accord avec l'affirmation de Musila selon laquelle la fiction de Kantai "élargit les horizons de la pratique littéraire populaire au Kenya " (Musila 2014 : 263), nous aimerions nous appuyer sur la conceptualisation faite par Barber du continuum entre "populaire» et "élitiste» pour nous pencher sur le lien qu'entretiennent les rédacteurs en chef de Kwani? avec le «canonique » et la mémoire culturelle et dégager certaines des tensions qui sous-tendent la volonté de Kahora de voir Kwani? promouvoir une forme de "mémoire populaire contre la mémoire officielle » (Stasio 2012).

19 Si Barber n'utilise pas le concept du « quotidien », l'ouvrage collectif dirigé par Newell et Okome place au premier plan la relation entre les arts populaires et « le quotidien » (« the everyday»), et insiste sur la façon dont la documentation, la construction et la transformation du quotidien représentent autant d'articulations culturelles collectives (Newell et Okome $2014: 3,14$ ). L'ouvrage montre ainsi que la culture populaire africaine constitue un lieu où se produisent les processus caractéristiques de la mémoire culturelle selon Assmann : la création d'une forme de savoir partagé à partir duquel un groupe forge son sentiment d'identité collective et sa relation au passé. Cependant, ces processus sont ici intimement liés aux expériences et aux activités quotidiennes qui contribuent à les produire. Pour Assmann, au contraire, la «mémoire culturelle se caractérise par la distance qui la sépare du quotidien » et la communication quotidienne propre à la mémoire communicative se caractérise par son "absence de forme " ( formlessness ») (Assmann 1995 : 129, 127). Ainsi, les catégories dégagées par Barber au sujet de la culture populaire africaine soulèvent toute une série de questions par rapport aux distinctions opérées par Assmann entre ces deux formes de mémoire: sa caractérisation des producteurs de mémoire communicative comme des «non-spécialistes » (126) d'une part, et la nécessité pour lui que la mémoire culturelle fasse l'objet d'une organisation et d'un « renforcement institutionnel » (131) d'autre part.

Cela étant dit, et sans perdre de vue ces tensions, le travail d'Assmann, en plaçant au premier plan le lien entre mémoire culturelle et communication quotidienne - même s'il n'en dégage pas toutes les nuances - offre un point d'entrée utile pour analyser la façon dont s'articule le travail du Kwani Trust par rapport au passé et à l'idée du " populaire ». Il permet de mettre en lumière la préoccupation de ce dernier de trouver de nouvelles formes littéraires à même d'exprimer une série de récits jusqu'alors absents des textes formels et officiels, en même temps qu'ils dotent ces récits, en les publiant sous leur forme populaire existante, d'une valeur culturelle. Ce que Barber appelle le « réseau de relations » (Barber 1987: 1) que l'on retrouve dans l'expression bourdieusienne de " réseau des relations d'échange ${ }^{18}$ » (Bourdieu [1992] 1995 : 230) et par lequel les textes sont produits et consacrés et dotés de valeur culturelle, est donc essentiel dans la position qu'occupe le Kwani Trust dans le continuum, ou peut-être à l'interface entre l'officiel / le canonique et le populaire. Si Musila considère que la revendication de Wainaina d'une « indépendance totale à l'égard des institutions universitaires et de mécénat " est compromise par les subventions que le Kwani Trust reçoit de la Fondation Ford et de la 
Fondation Doen (Musila 2014 : 251), nous aimerions quant à nous avancer l'idée que Wainaina n'a aucune intention de positionner Kwani? à l'extérieur de ces processus de légitimation, et insister sur le fait que l'esthétique recherchée par la revue puise son inspiration dans la culture populaire kenyane, que les institutions culturelles en place - de l'université au gouvernement, en passant par les financeurs étrangers de l'industrie culturelle - avaient perdue de vue et ne légitimaient pas. Cette idée se retrouve dans la rubrique "About Us» du site internet du Kwani Trust en 2005, dans laquelle les rédacteurs en chef se félicitaient du succès et des 5000 exemplaires vendus, dans un pays où, selon les enseignants et les éditeurs, il n'existait pas de culture de la lecture :

[...] voici ce dont nous nous sommes aperçus : l'intelligentsia littéraire, les éditeurs africains et les fondateurs de projets littéraires ont perdu de vue une nouvelle génération d'Africains qui en ont assez qu'on leur parle de haut et qui cherchent à faire sens du monde déroutant qui les entoure - et à être légitimés par l'écrit ${ }^{19}$. (Kwani Trust 2005)

21 Il nous semble plutôt, comme nous l'avons montré ailleurs (Wallis 2016), que le Kwani Trust était conscient des multiples processus de légitimation par lesquels les textes produisent du sens, et ont adopté leur ligne éditoriale en conséquence. Lorsque Kahora écrit que certains récits sont invisibles dans les "textes publics officiels et formels " (Kahora 2007 : i), il fait référence non seulement aux silences des médias ou aux formes de savoir sur lesquelles l'État exerce une forme de contrôle, mais aussi aux façons dont l'orchestration de l'amnésie nationale a empêché qu'une variété de récits voient le jour dans des productions culturelles légitimées comme de la littérature et dans des productions de savoir légitimées comme de l'histoire. Lorsqu'il réfléchit à sa volonté de recouvrer une forme de mémoire de collective par l'écriture, Kantai souligne que les tribunes médiatiques "n'étaient pas seulement limitées, elles étaient aussi très éphémères » tandis que la « fiction » et sa publication dans Kwani? semblait garantir « une certaine mesure de pérennité » (Kantai 2012). Comme nous l'avons montré dans un article publié dans Wasafiri en 2016, la forme imprimée devait jouer un rôle central dans l'idée de pérennité et de légitimation. Kahora souligne ainsi que l'idée de rendre compte de l'élection présidentielle de 2007 dans le cinquième numéro de la revue est née de la conviction, partagée avec Wainaina, qu'il fallait garder des traces écrites qui pourraient être consultées à l'avenir : « Peut-être que tout n'y sera pas dit, mais il faut que nous le consignions dans un livre ${ }^{20} »$ (Kahora 2011).

Du fait de la légitimité dont jouissaient la littérature et les récits partiaux dans l'espace national, le Kwani Trust a délibérément cherché à être reconnu dans l'« espace littéraire mondial ${ }^{21} »$ (Casanova [1999] 2004 : xii). Cependant, à côté de cette insistance à nouer des relations avec et à être reconnue par des institutions littéraires continentales, aux ÉtatsUnis et en Grande-Bretagne, le Kwani Trust, en tant qu'organisation, cherche aussi à entretenir des relations avec l'institution universitaire et le gouvernement kenyans, et ce malgré les débats houleux qui l'opposèrent à ses débuts aux universitaires et aux journalistes locaux. Dans un article paru en 2013 dans The Nation, Kingwa Kamencu observait qu'il était « rafraîchissant » de voir une partie des festivités organisées pour les dix ans de la revue se tenir dans des "institutions universitaires", à l'université de Nairobi et à l'université Kenyatta et que cela témoignait des «rapports cordiaux qu'entretenaient aujourd'hui d'anciens groupes rivaux » (Kamencu 2013). Si les relations entre le Kwani Trust et l'institution universitaire ont évolué avec le temps, les conférences et discussions publiques au sein de l'université de Nairobi ont toujours été au programme des festivals (Kwani Litfest) organisés par le collectif : Chimamanda Ngozi 
Adichie, Doreen Baingana, Ishmael Beah, M.G. Vassanji et Binyavanga Wainaina ont ainsi pris part à une discussion organisée lors de la première édition du festival en 2006 et qui se tenait dans la Taifa Hall de l'université de Nairobi. Dans un éditorial intitulé « Editor's Rant » et publié dans le premier numéro de la revue, Wainaina se plaint du fait que ni lui ni Yvonne Adhiambo Owuor n'ont reçu de reconnaissance du ministère de la Culture pour avoir remporté le prix Caine. Ce texte témoigne peut-être de la volonté des rédacteurs en chef de Kwani? de se positionner en dehors des structures officielles de légitimation, qu'il critique : sur un mode dialogique, le texte avance que Kalamashaka et Richard Onyango «devraient être reconnus pour le travail pionnier qu'ils accomplissent» et pose la question suivante : "doit-on encore s'attendre à ce que les éternels chefs de chœur et chanteurs de louanges soient la priorité du programme culturel du gouvernement?» (Wainaina 2003b : 233). Il semble toutefois que cela témoigne d'un désir, plutôt que d'un rejet, de la reconnaissance par l'État et de la légitimation qui y est associée :

Je donne peut-être l'impression de rechercher la gloire ou les médailles ou je ne sais quoi. Non. Je suis simplement troublé que le gouvernement refuse de prendre au sérieux ce que nous faisons. Il s'agit après tout de NOTRE gouvernement. Comme beaucoup de la nouvelle génération, je suis troublé que nos efforts comptent si peu 22. (Wainaina 2003b : 233)

Certaines des dynamiques que nous venons de décrire semblent aller à l'encontre de certaines caractéristiques dégagées par Barber à propos des "arts populaires ». La volonté de consigner et documenter le passé, de s'appuyer sur des instances de légitimation qui permettent la création de quelque chose de pérenne, qu'il sera possible de consulter à l'avenir (Kahora 2011) ne semble pas entrer dans le cadre de « l'esthétique de l'effet immédiat » mise en avant par Barber (1987 : 45). De la même façon, il semble que les conventions sur lesquelles s'appuie Kwani? pour « construire du sens et communiquer avec son public » sont plutôt que ne sont pas légitimées et reconnues publiquement. La revue littéraire imprimée en tant que forme qui occupe une place importante dans l'histoire de l'édition en Afrique et dans l'espace littéraire mondial depuis le XIX siècle a toujours permis d'incorporer une variété de genres et d'artefacts issus de la culture populaire (Patten 2006: 360). Et pourtant, il nous semble que le champ d'expression insaisissable des «arts populaires" théorisé par Barber est un concept clé pour comprendre les textes publiés par le Kwani Trust, et plus particulièrement son rapport à la mémoire et à l'histoire. Barber a montré que la nature syncrétique des arts populaires est ancrée dans une forme de négociation culturelle, quand elle décrit les producteurs d'arts populaires comme des "passeurs culturels » qui, au sein de la société, regardent dans les deux directions à la fois et s'appuient sur des éléments issus de « deux ensembles de ressources culturelles différents » (Barber 1987 : 39). Une lecture du programme du festival Kwani Litfest de 2006 témoigne de la position de passeur culturel occupée par le Kwani Trust: au programme de la soirée d'ouverture du festival le 14 décembre on trouvait l'artiste Richard Onyango, dont les mémoires («Life and Times ») étaient parus dans Kwani? 1 et une session live du DJ Ntone Edjabe, rédacteur en chef de la revue Chimurenga (Afrique du Sud), mais aussi la conférence prévue (mais finalement annulée) du ministre des Affaires étrangères Rafael Tuju. Le 15 décembre, une série de conférences ouvertes au public se tenait à l'université de Nairobi et réunissait Chimamanda Ngozi Adichie, Doreen Baingana et Binyavanga Wainaina autour du thème de la place des écrivains africains sur la scène mondiale ("African Writers on the Global Stage») (Pambazuka 2006). Ainsi, le Kwani Trust se présentait comme un passeur culturel dont le regard est tourné vers différentes directions - vers les espaces culturels officiels et non 
officiels au Kenya, mais aussi vers l'extérieur, vers d'autres éditeurs du continent et audelà, vers l'« espace littéraire mondial ». Si les définitions que donne Barber des "arts populaires » permettent de problématiser les distinctions établies par Assmann entre mémoire communicative et mémoire culturelle, son travail semble proposer un modèle grâce auquel il est possible d'envisager ces deux notions non comme des catégories fixes, mais plutôt comme des champs d'expression et ainsi d'envisager un continuum entre mémoire communicative et mémoire culturelle, sur lequel, comme nous l'avons montré, le Kwani Trust est à même d'intervenir en tant que passeur culturel dans l'espace de ce que nous appelons la « mémoire populaire ».

\section{BIBLIOGRAPHIE}

Assmann, Jan, 1995, « Memory and Cultural Identity », New German Critique, n 65, p. 125-133.

Barber, Karin, 1987, « Popular Arts in Africa », African Studies Review, vol. 30, nº 3, p. 1-78.

Bourdieu, Pierre, 1995, The Rules of Art: Genesis and Structure of the Literary Field, trad. Susan Emanuel, Stanford, Stanford University Press.

Bourdieu, Pierre, [1992] 1998, Les Règles de l'art. Genèse et structure du champ littéraire, Paris, Seuil. Branch, Daniel, 2011, Kenya: Between Hope and Despair, 1963-2011, Londres, Yale University Press.

Casanova, Pascale, 2004, The World Republic of Letters, trad. M. B. DeBevoise, Cambridge, MA, Harvard University Press.

Casanova, Pascale, [1999] 2008, La République mondiale des lettres, Paris, Seuil.

Clough, Marshall S., 2003, « Mau Mau \& the Contest for Memory », dans E.S. A. Odhiambo et J. Lonsdale (dir.), Mau Mau \& Nationhood: Arms, Authority \& Narration, Oxford, James Currey, p. 251-267.

GoDown Arts Centre, 2016, « History », [En ligne], http://www.thegodownartscentre.com/ index.php/aboutthegodown/history, consulté le 15 juillet 2019.

Halbwachs, Maurice, 1992, On Collective Memory, trad. Lewis A. Coser, Chicago, University of Chicago Press.

Hughes, Lotte, 2011, " “Truth be Told”: Some Problems with Historical Revisionism in Kenya », African Studies, vol. 70, $\mathrm{n}^{\circ}$ 2, p. 182-201.

Hughes, Lotte, Coombes, Annie E. et Karega-Munene, 2011, «Introduction », African Studies, vol. 70, $\mathrm{n}^{\circ} 2$, p. 175-181.

Kahora, Billy, 2005, « Editorial », Kwani? 03, p. 6-7.

Kahora, Billy, 2007, « Editorial », Kwani? 04, p. i-iii.

Kahora, Billy (dir.), 2008a, Kwani? 05 Part 1: Maps and Journeys, Nairobi, Kwani Trust.

Kahora, Billy (dir.), 2008b, Kwani? 05 Part 2: Revelation and Conversation, Nairobi, Kwani Trust.

Kahora, Billy, 2008c, The True Story of David Munyakei, Nairobi, Kwani Trust. 
Kahora, Billy, 2011, Interview by Kate Haines, Nairobi, 24 mai.

Kahora, Billy, et Wainaina, Binyavanga (éds.), 2005, Kwani? 03, Nairobi, Kwani Trust.

Kalamashaka, 2003, « Moto », Kwani? 01, p. 53-54.

Kamencu, Kingwa, 2013, « "Literary gangsters” take stock 10 years on », Nation, [En ligne], http:// www.nation.co.ke/lifestyle/weekend/-Literary-gangsters--take-stock-10-yearson/-/1220/2101940/-/ap74fr/-/index.html, consulté le 15 jullet 2019.

Kantai, Parselelo, 2004, « Comrade Lemma \& the Black Jerusalem Boys Band », dans Kwani? 02, éd. B. Wainaina et E. Kalondo, p. 208-223.

Kantai, Parselelo, 2006, « Death of the Kenya Dream? », The East African, 31 juillet, [En ligne], http://allafrica.com/stories/200607311009.html, consulté le 15 jullet 2019.

Kantai, Parselelo, 2011, Interview par Kate Haines, Nairobi, 25 mai.

Kantai, Parselelo, 2012, « Writing history’s silences », Kunapipi, vol. 34, nº 1.

Kasaya, Eva, 2010, Tale of Kasaya, Nairobi, Kwani Trust.

Ketebul Music, 2016, « About Us », [En ligne], http://www.ketebulmusic.org/about-us/, consulté le 15 jullet 2019.

Kisia, Andia, 2003, « A Likely Story », Kwani? 01, éd. Binyavanga Wainaina, p. 70-85.

Kwani Trust, 2005, « About Us », [En ligne, archive], http://web.archive.org/ web/20051219081607/http://www.kwani.org/about\%20us.htm, consulté le 15 jullet 2019.

Kwani Trust, 2016, « Tale of Kasaya », [En ligne, archive], https://web.archive.org/ web/20170818081418/http://kwani.org/publication/kwani-series/164/tale_of_kasaya.htm, consulté le 15 juillet 2019.

Musila, Grace, 2014, " Archives of the Present in Parselelo Kantai's Writing », dans S. Newell et O. Okome (dir.), Popular Culture in Africa: The Episteme of the Everyday, Abingdon, Routledge.

Muthee, Joseph, 2006, Kizuizini, Nairobi, Kwani Trust.

Mwangi, Macharia, 2015, « Publishing Outposts on the Kenyan Literary Landscape: A Critique of Busara, Mũtiiri and Kwani? », Thèse de doctorat, Université de Nairobi.

Newell, Stephanie, 2000, Ghanaian Popular Fiction: "Thrilling Discoveries in Conjugal Life" \& Other Tales , Athens, OH, Ohio University Press.

Newell, Stephanie et Okome, Onookome, 2014, « Introduction », dans S. Newell et O. Okome (dir.), Popular Culture in Africa: Episteme of the Everyday, New York, Routledge.

Njogu, Kimani (dir.), 2009a, Healing the Wound: Personal Narratives About the 2007 Post-Election Violence in Kenya, Nairobi, Twaweza Communications.

Njogu, Kimani, 2009b, « Introduction », dans K. Njogu (dir.), Healing the Wound: Personal Narratives About the 2007 Post-Election Violence in Kenya, Nairobi, Twaweza Communictions, p. 1-12.

Njogu, Kimani, 2011a, Interview par Kate Haines, Nairobi, 29 septembre.

Njogu, Kimani, (dir.), 2011b, Defining Moments: Reflections on Citizenship, Violence and the 2007 General Elections in Kenya, Nairobi, Twaweza Communications.

Nyairo, Joyce, 2005, « Zilizopendwa: Kayamba Afrika's use of cover versions, remix and sampling in the (re)membering of Kenya ", African Studies, vol. 64, $n^{\circ}$ 1, p. 29-54. 
Nyairo, Joyce, 2007, « "Modify”: Jua Kali as a Metaphor for Africa's Urban Ethnicities and Cultures ", dans J. Ogude et L. Nyariki (dir.), Urban Legends, Colonial Myths: Popular Culture and Literature in East Africa, Trenton, NJ, Africa World Press, Inc.

Nyairo, Joyce, 2011, Interview par Kate Haines. Nairobi, 30 septembre.

Nyairo, Joyce, 2015, Kenya@50: Trends, Identities and the Politics of Belonging. Nairobi: CONTACT ZONES NRB.

Odhiambo, E.S Atieno, 1987, « Democracy and the Ideology of Order in Kenya », dans M. Schatzberg (dir.), The Political Economy of Kenya, New York, Praeger, p. 177-201.

Odhiambo, Tom, 2011, « Kwani? and the Imaginations Around Re-Invention of Art and Culture in Kenya », dans J. Ogude, G. Musila et D. Ligaga (dir.), Rethinking Eastern African Literary and Intellectual Landscapes, Trenton, NJ, Africa World Press.

Ogude, James, 2007, " "The Cat that ended up eating the Homestead Chicken": Murder, Memory and Fabulization in D. O. Misiani's Dissident Music ", dans J. Ogude et J. Nyairo (dir.), Urban Legends, Colonial Myths: Popular Culture and Literature in East Africa, Trenton, NJ, Africa World Press, Inc, p. 173-200.

Ogude, James, Musila, Grace et Ligaga, Dina, 2011a, « Introduction », dans J. Ogude, G. Musila et D. Ligaga (dir.), Rethinking Eastern African Literary and Intellectual Landscapes, Trenton, NJ, Africa World Press, p. vii-xxvi.

Ogude, James, Musila, Grace et Ligaga, Dina (dir.), 2011b, Rethinking Eastern African Literary and Intellectual Landscapes, Trenton, NJ, Africa World Press.

Ogude, James, et Nyairo, Joyce (dir.), 2007, Urban Legends, Colonial Myths: Popular Culture and Literature in East Africa, Trenton, NJ, Africa World Press.

Ojwang, Dan, 2009, « Kenyan Intellectuals and the Political Realm: Responsibilities and Complicities », Africa Insight, vol. 39, n 1, p. 22-38.

Owuor, Yvonne Adhiambo, 2013, Dust, Nairobi, Kwani Trust.

Pambazuka, 2006, « KWANI LITFEST 2006: Ideas...Words...Markets », [En ligne], http:// www.pambazuka.org/resources/africa-ideas...words...markets, consulté le 15 juillet 2019.

Patten, Robert, 2006, «When is a Book not a Book? », dans D. Finkelstein et A. McCleery (dir.), The Book History Reader, Abingdon, Routledge, p. 354-368.

Stasio, Frank, 2012, « Reimagining Kenya's Future through Literature: Interview with Billy Kahora ", WUNC: North Carolina Public Radio, 20 janvier.

Wainaina, Binyavanga, 2003a, « Editorial », Kwani? 01, edited by Binyavanga Wainaina, Nairobi, Kwani Trust.

Wainaina, Binyavanga (dir.), 2003b, Kwani? 01, Nairobi, Kwani Trust.

Wainaina, Binyavanga, 2014, Interview par Kate Haines, Londres, 7 mars.

Wainaina, Binyavanga et Kalondo, Ebba (éds.), 2004, Kwani? 02, Nairobi, Kwani Trust.

Wallis, Kate, 2016, « How Books Matter: Kwani Trust, Farafina, Cassava Republic Press and the Medium of Print », Wasafari, vol. 31, $\mathrm{n}^{\circ} 4$ (Special Issue: Under Pressure: Print Activism in the 21st Century), p. 39-46. 


\section{NOTES}

1. $\mathrm{NdT}$ : sauf mention contraire, toutes les citations sont traduites par la traductrice.

2. «[...] accidents of chance, miscellaneous imperfections (though human) and any other inconvenient facts. »

3. « He had had enough of his brains being picked and the pickings fashioned into tales that were no longer recognizable to him who had told them. »

4. " high degree of nonspecialization, reciprocity of roles, thematic instability, and disorganization. »

5. «[...] continues to cloak myriad subjects such as Moi's tyrannical rule and legacy, political assassinations, detention and torture during the Kenyatta and Moi eras, land grabbing by elites, corruption on a lavish scale, and so on. »

6. On peut signaler ici que Joyce Nyairo, qui avait enseigné pendant plus de vingt ans à l'université Moi, au sein du département d'études littéraires, théâtrales et cinématographiques, et dont la recherche portait sur les liens entre musique populaire et mémoire, devint en 2007 chargée de mission (« programme officer ») auprès de l'antenne de la Fondation Ford en Afrique de l'est. La Fondation Ford, une fondation privée dont le siège est à New York et dont la mission est de promouvoir la justice sociale (Ford Foundation 2016), avait accordé au Kwani Trust les fonds lui permettant d'installer ses bureaux à Nairobi, et de lancer la revue Kwani? en 2003. Au cours de ses cinq années passées à la Ford Fondation, Nyairo a développé des passerelles entre sa recherche universitaire et la création artistique en initiant et soutenant une série de projets et d'organisations dans le domaine des médias et de la mémoire, qu'elle a elle-même baptisés « projets liés à la mémoire culturelle » (Nyairo 2015, 2011). Parmi ces projets, on peut citer la série de Ketebul Music intitulée "Retracing Popular Music", un projet artistique à l'Institut Goethe intitulé "Amnesia» qui s'intéressait aux conséquences culturelles de la perte de mémoire culturelle, deux nouvelles séries de biographies sur les femmes et les sportifs. Ce fut également elle qui encouragea le Kwani Trust à regarder vers le passé en soutenant le festival (Kwani LitFest) que celui-ci organisa en 2010 sur le thème "Tell Us What Happened » et qui comprenait une série de conversations entre écrivains kényans de différentes générations (Nyairo 2011).

7. «A collective narrative on what we were before, and what we became, during the epochal first 100 days of 2008. »

8. «People who import their histories are doomed by the failure of their own imaginations. »

9. «[...] behind the closed doors of the Kenyan urban family to reveal spaces that we all recognize but refuse to acknowledge. »

10. «Tell me who would be the first to say the word harambee? Dedan Kimathi or Mzee Jomo Kenyatta?» $[\mathrm{NdT}]$ : «Harambee » est un terme swahili qui signifie "tirons tous ensemble », ce terme est devenu au moment de l'indépendance la devise du pays.

11. [NdT] Les matatus sont des minibus de transport collectif.

12. " And though the facts matter, it is the inducement of commonality, a feeling that I share enough with a stranger to care about a shared narrative, to believe the 
unbelievable, the improbable version; that is what makes me feel more Kenyan than anything else.»

13. Ce sont aussi ces lieux que Grace Musila appelle, dans son ouvrage A Death Retold in Truth and Rumour, les " "tribunaux" parallèles du ouï-dire" (Musila 2015 : 111) qui se développent quand la "rumeur " permet d'analyser et de combler les lacunes de la science et de la loi, et construisent ainsi une « version de la vérité » dotée de bien plus de légitimité et de crédibilité.

14. «[...] one of the very few vehicles... to negotiate complex issues of power away from the watchful eye of the state. »

15. «[...] how to make what they were writing reflect the language of the Kenyan street, the Kenyan shamba, the Kenyan bar, and of course the language of the Kenyan family. "

16. «[...] will not be donated to us from the corridors of the university; or from the ministry of culture, or by The French Cultural Centre. It will come from the individual creations of thousands of creative people. »

17. «[...] combine elements from the traditional and the metropolitan cultures in unprecedented conjunctures, with the effect of radical departure from both. "

18. La référence de la citation en français est la suivante : Bourdieu [1992] $1998: 373$.

19. "What we have found is that the literary intelligentsia, together with African publishers and founders of literary projects have lost touch with a new generation of Africans who are sick of being talked down to; who are seeking to understand the bewildering world around them-to be validated in print. »

20. «It might not say everything, we just need to actually record this in a book. »

21. La référence de la citation originale en français est la suivante : Casanova [1999] 2008 : xi.

22. « Now I may be sounding much like I want fame or fortune or medals or something. No - I am simply disturbed by the refusal of our government to take what we are doing seriously. After all, they are OUR government. Like many of a new generation, I am disturbed by the way our efforts seem not to matter. »

\section{RÉSUMÉS}

Cet article s'intéresse aux diverses façons dont la revue Kwani? a créé de nouveaux lieux et de nouvelles formes d'expression de la mémoire culturelle. Nous montrerons que la ligne éditoriale et les textes des principaux membres du réseau littéraire que constitue le Kwani Trust, et dont font partie Andia Kisia, Parselelo Kantai, Billy Kahora et Yvonne Adhiambo Owuor, témoignent d'une préoccupation particulière pour l'historiographie et pour les façons dont la mémoire et l'histoire se construisent et prennent forme en tant que textes. Nous replacerons ces questions dans le contexte plus large de la production culturelle au Kenya, notamment celle financée par les donateurs étrangers, et qui s'intéresse, elle aussi, aux questions d'histoire et de mémoire. Il s'agira de rendre compte des différentes manières dont ces écrivains abordent et explorent les questions suivantes: Où se situe la production du savoir historique au Kenya? Qui produit 
l'histoire et pour qui ? Quels sont les liens qui unissent mémoire, littérature et matérialité ? Nous ferons dialoguer la notion de mémoire collective, élaborée par l'égyptologue allemand Jan Assmann et constituée selon lui de la « mémoire culturelle » et de la « mémoire communicative », avec les définitions que propose Karin Barber des «arts populaires» (Barber 1987) afin de montrer comment ce groupe d'écrivains s'attache à la construction d'une mémoire populaire.

This article explores the ways in which the Kwani? journal has opened up new forms and spaces for cultural memory. It argues that the editorial agenda of, as well as the work of some of the most prominent voices associated with Kwani Trust as a literary network (including Andia Kisia, Parselelo Kantai, Billy Kahora and Yvonne Adhiambo Owuor), reflects a particular preoccupation with historiography-or the ways in which memory and history are constructed and take form as texts. Setting this in the context of the wider engagement of donor-funded cultural production in Kenya with questions of history and memory in this period, it examines the different ways that these writers confront and explore questions around where knowledge about Kenya's past is located, who history is produced by and for, and memory's relationship to literature and material form. Building on and problematizing Jan Assmann's framing of collective memory as made up of "cultural memory" and "communicative memory," and putting this in dialogue with Karin Barber's definitions of "popular arts" (Barber 1987), it characterizes this group of writers as engaged in the construction of popular memory.

\section{INDEX}

Keywords : Kwani Trust, popular arts, cultural memory, Kenya, history, contemporary African literature

Mots-clés : Kwani Trust, arts populaires, mémoire culturelle, Kenya, histoire, littérature africaine contemporaine

\section{AUTEURS}

\section{KATE WALLIS}

University of Exeter 